\title{
Association of renal function with clinical parameters and conditions in a longitudinal population-based epidemiological study
}

\author{
TAKUYA SUMI ${ }^{1}$, MITSUTOSHI OGURI ${ }^{2}$, TETSUO FUJIMAKI ${ }^{3}$, HIDEKI HORIBE $^{4}$, KIMIHIKO KATO $^{5}$, \\ KOTA MATSUI $^{6}$, ICHIRO TAKEUCHI ${ }^{7,8}$, TOYOAKI MUROHARA ${ }^{1}$ and YOSHIJI YAMADA ${ }^{8,9}$ \\ ${ }^{1}$ Department of Cardiology, Graduate School of Medicine, Nagoya University, Nagoya, Aichi 466-8560; \\ ${ }^{2}$ Department of Cardiology, Kasugai Municipal Hospital, Kasugai, Aichi 486-8510; ${ }^{3}$ Department of Cardiovascular Medicine, \\ Inabe General Hospital, Inabe, Mie 511-0428; ${ }^{4}$ Department of Cardiovascular Medicine, Gifu Prefectural Tajimi Hospital, \\ Tajimi, Gifu 507-0042; ${ }^{5}$ Department of Internal Medicine, Meitoh Hospital, Nagoya, Aichi 465-0025; ${ }^{6}$ Department of \\ Biostatistics, Graduate School of Medicine, Nagoya University, Nagoya, Aichi 464-8601; ${ }^{7}$ Department of \\ Computer Science, Graduate School of Engineering, Nagoya Institute of Technology, Nagoya, Aichi 466-8555; \\ ${ }^{8}$ Core Research for Evolutionary Science and Technology (CREST), Japan Science and Technology Agency, \\ Kawaguchi, Saitama 332-0012; ${ }^{9}$ Department of Human Functional Genomics, \\ Life Science Research Center, Mie University, Tsu, Mie 514-8507, Japan
}

Received October 21, 2016; Accepted November 28, 2016

DOI: $10.3892 /$ br.2016.831

\begin{abstract}
The aim of the present study was to examine the association of renal function with clinical parameters and conditions in the general population. Study subjects comprised 6,027 community-dwelling individuals who were recruited to the Inabe Health and Longevity Study: A longitudinal genetic epidemiological study of atherosclerotic, cardiovascular and metabolic diseases. The cutoff value, which was used to divide the subjects into those with normal and those with low estimated glomerular filtration rate (eGFR), was $60 \mathrm{ml} / \mathrm{min} / 1.73 \mathrm{~m}^{2}$. Bonferroni's correction was applied to establish the statistical significance of the association. Longitudinal analysis using the generalized linear mixed-effect model, following adjustments for age and gender, revealed that the eGFR was significantly associated $(\mathrm{P}<0.0017)$ with serum levels of triglycerides, low-density lipoprotein cholesterol, uric acid, blood glycosylated hemoglobin content, fasting plasma glucose and body mass index. These parameters decreased curvilinearly with increases in eGFR. Furthermore, eGFR correlated positively with serum levels of high-density
\end{abstract}

Correspondence to: Dr Mitsutoshi Oguri, Department of Cardiology, Kasugai Municipal Hospital, 1-1-1 Takaki-cho, Kasugai, Aichi 486-8510, Japan

E-mail: oguricap0909@gmail.com

Key words: estimated glomerular filtration rate, longitudinal study, diabetes mellitus, hypertension, dyslipidemia, hyperuricemia, obesity lipoprotein (HDL) cholesterol. Longitudinal analysis using the generalized estimating equation following adjustment for age and gender indicated a significant association $(\mathrm{P}<0.0024)$ between eGFR and prevalence of hypertension, type 2 diabetes mellitus, hypo-HDL cholesterolemia, hyperuricemia and obesity. Thus, low eGFR results in detrimental effects on various clinical parameters and conditions, resulting in increased risk of hypertension, dyslipidemia, type 2 diabetes mellitus, hyperuricemia and obesity.

\section{Introduction}

Chronic kidney disease (CKD) is associated with various cardiovascular diseases and increased healthcare costs (1). The glomerular filtration rate (GFR) diminishes with age by 0.6 to $1.1 \mathrm{ml} / \mathrm{min} /$ year, and the estimated prevalence of CKD is $\sim 13 \%$ in the general population in Japan (2). In addition, epidemiologic studies reported that development of CKD leads to progression of atherosclerosis even during the initial stage $(3,4)$.

Diabetes mellitus (DM) is an established risk factor for the development of CKD (5). A large cohort study reported a strong association between fasting plasma glucose (FPG) level and renal function even in subjects without DM (6). Furthermore, hypertension and/or dyslipidemia frequently coexist with CKD, which are often associated with marked renal impairment $(7,8)$. Given that risk factor clustering is a strong predictor of future progression of renal dysfunction, it is important to select appropriate therapeutic strategies that take into consideration risk stratification and control of multiple risk profiles. However, the temporal association between the above-mentioned risk factors and renal function with aging remains unclear in Japanese patients. The present large-scale longitudinal study was designed to clarify the association 
of renal dysfunction with a multitude of clinicopathological parameters and conditions, and to define age-associated changes in these parameters in the general population.

\section{Materials and methods}

Study subjects. A total of 6,027 community-dwelling individuals were recruited to the Inabe Health and Longevity Study: A longitudinal epidemiological study of atherosclerosis, and cardiovascular and metabolic diseases (9-12). The subjects were recruited from among individuals who visited the health care center of Inabe General Hospital (Inabe, Japan) for their annual health checkup, and who were followed up annually. For all participants registered between March 2010 and September 2012, clinical examination data obtained from April 2003 to March 2014 (11 years) were entered into a database. For individuals with two or more medical checkups per year, data from one time point for each year were entered, so that each subject had one set of health data for each year they had attended the clinic. In general, the study participants had undergone one to 11 clinical examinations, and the mean follow-up period was 5 years.

The study protocol was complied according to the Declaration of Helsinki and was approved by the Committees on the Ethics of Human Research of Mie University Graduate School of Medicine (Tsu, Japan) and Inabe General Hospital. Written informed consent was obtained from each subject.

Definition of clinical conditions. The estimated GFR (eGFR) was calculated using a simplified equation derived from that in the Modification of Diet in Renal Disease Study and proposed by the Japanese Society of Nephrology: eGFR $\left(\mathrm{ml} / \mathrm{min} / 1.73 \mathrm{~m}^{2}\right)=194 \times$ [age (years) $]^{-0.287} \times$ [serum creatinine $(\mathrm{mg} / \mathrm{dl})]^{-1.094} \times$ [0.739 for females] (13). Low eGFR represented values $<60 \mathrm{ml} / \mathrm{min} / 1.73 \mathrm{~m}^{2}$, based on the National Kidney Foundation-Kidney Disease Outcomes Quality Initiative (1). Thus, 592 subjects were diagnosed with low eGFR. The eGFR of the control subjects $(n=4,928)$ was $\geq 60 \mathrm{ml} / \mathrm{min} / 1.73 \mathrm{~m}^{2}$. Subjects with hypertension either had a systolic blood pressure (BP) $\geq 140 \mathrm{mmHg}$ or diastolic BP $\geq 90 \mathrm{mmHg}$ (or both) or were currently on antihypertensive medication. DM was defined as either FPG $\geq 6.93 \mathrm{mmol} / \mathrm{l}$ or blood hemoglobin Alc content $\geq 47.5 \mathrm{mmol} / \mathrm{mol}$ or current use of glucose-lowering agents. Hypertriglyceridemia was defined as either serum triglyceride concentration $\geq 1.65 \mathrm{mmol} / \mathrm{l}$ or use of antidyslipidemic medications for hypertriglyceridemia. Hypo-high-density lipoprotein (HDL) cholesterolemia was defined as serum HDL-cholesterol concentration $<1.04$ $\mathrm{mmol} / \mathrm{l}$. Hyper-low-density lipoprotein (LDL) cholesterolemia was defined as either serum LDL-cholesterol concentration $\geq 3.64 \mathrm{mmol} / \mathrm{l}$ or current treatment with antidyslipidemic agents for hyper-LDL-cholesterolemia. Hyperuricemia was defined as serum concentration of uric acid $>416 \mu \mathrm{mol} / \mathrm{l}$ or current treatment with uric acid-lowering medication. Obesity was defined as body mass index (BMI) $\geq 25 \mathrm{~kg} / \mathrm{m}^{2}$ and BMI of $<25 \mathrm{~kg} / \mathrm{m}^{2}$ for the control individuals, based on the BMI criteria of obesity for Japanese and Asian populations (14).

Statistical analysis. Categorical data were compared between two groups using the $\chi^{2}$ test. The distribution of continuous variables was examined by Shapiro-Wilk test. Comparison between two groups was conducted using the unpaired Student's t-test (for variables with normal distribution) or by Mann-Whitney U test (for variables with skewed distribution). The association between eGFR and various clinical parameters and conditions was examined based on a 5-year longitudinal cohort study. Longitudinal changes in clinical parameters were analyzed with a generalized linear mixed-effect model (15) after adjustment for age and gender. Correlations of quantitative data were examined by simple regression analysis with fitting to a straight line or quadratic curve. Longitudinal changes in the prevalence of hypertension, type $2 \mathrm{DM}$, hypertriglyceridemia, hypo-HDL-cholesterolemia, hyper-LDL-cholesterolemia, hyperuricemia and obesity were analyzed with the generalized estimating equation (16) after adjustment for age and gender. Subjects with eGFR $<40$ or $>100 \mathrm{ml} / \mathrm{min} / 1.73 \mathrm{~m}^{2}$ were excluded from analysis, as the number of such subjects was small ( 40 with eGFR $<40 \mathrm{ml} / \mathrm{min} / 1.73 \mathrm{~m}^{2} ; 408$ with eGFR $>100 \mathrm{ml} / \mathrm{min} / 1.73 \mathrm{~m}^{2}$ ). Age-associated changes in the prevalence of hypertension, type $2 \mathrm{DM}$, hypertriglyceridemia, hypo-HDL-cholesterolemia, hyper-LDL-cholesterolemia, hyperuricemia and obesity were compared between subjects with low eGFR and the control subjects using a generalized estimating equation. To compensate for multiple comparisons of variables, Bonferroni's correction was applied for statistical significance of association. The significance levels were therefore as follows: $\mathrm{P}<0.0023$ (0.05/22 tests) in Table I; $\mathrm{P}<0.0017$ ( $0.05 / 30$ tests) in Table II; $\mathrm{P}<0.0024$ (0.05/21 tests) in Table III; $\mathrm{P}<0.0025$ ( $0.05 / 20$ tests) in Table IV; and $\mathrm{P}<0.0036$ (0.05/14 tests) in Table V. Statistical analysis was performed using the R software version 3.2.1 (the R Project for Statistical Computing; http://ww1.rproject.org/) and JMP version 5.1 software (SAS Institute, Inc., Cary, NC, USA).

\section{Results}

Patient characteristics. The baseline characteristics of the study subjects are presented in Table I. The prevalence of low eGFR was $10.7 \%$ (men, $12.3 \%$; women, $8.7 \%$ ). In subjects with low eGFR, age, proportion of men, BMI, systolic and mean $\mathrm{BP}$, serum concentrations of triglycerides, uric acid, blood urea nitrogen, and creatinine, FPG level, and blood hemoglobin A1c content were significantly increased $(\mathrm{P}<0.0023)$ when compared with the control subjects. Whereas serum concentration of HDL-cholesterol, and blood hemoglobin, hematocrit, and platelet count were lower in subjects with low eGFR versus the control subjects. Since the recruitment period and follow-up period were different among subjects, it was difficult to evaluate the initial eGFR on the inclusion point. Therefore, we calculated the mean eGFR at each year (Fig. 1). No differences were identified between the mean eGFR among years examined ( $\mathrm{P}=0.4433$, one-way analysis of variance).

Longitudinal analysis of the associations between eGFR and clinical parameters. After adjustments for age and gender, longitudinal analysis using the generalized linear mixed-effect model showed a significant association $(\mathrm{P}<0.0017)$ between eGFR and serum concentrations of triglycerides, HDL-cholesterol, LDL-cholesterol and uric acid, blood hemoglobin A1c content, FPG level and BMI using data from all 
Table I. Characteristics of study subjects: Cross-sectional analysis in March 2014.

\begin{tabular}{|c|c|c|c|c|}
\hline Parameter & All, $n=5520$ & Low eGFR, n=592 & Control, $n=4928$ & P-value \\
\hline Age (years) & $54.1 \pm 12.8$ & $66.4 \pm 9.3$ & $52.8 \pm 12.4$ & $<0.0001$ \\
\hline Male $(\%)$ & 55.3 & 64.2 & 55.1 & $<0.0001$ \\
\hline Body mass index $\left(\mathrm{kg} / \mathrm{m}^{2}\right)$ & $23.0 \pm 3.4$ & $23.6 \pm 3.2$ & $22.9 \pm 3.4$ & $<0.0001$ \\
\hline Current or former smoker $(\%)$ & 46.6 & 43.4 & 47.0 & 0.0980 \\
\hline Systolic BP (mmHg) & $120.8 \pm 16.0$ & $125.2 \pm 16.1$ & $120.3 \pm 15.8$ & $<0.0001$ \\
\hline Diastolic BP (mmHg) & $74.9 \pm 12.1$ & $76.0 \pm 12.0$ & $74.7 \pm 12.1$ & 0.0060 \\
\hline Mean BP (mmHg) & $90.2 \pm 12.5$ & $92.4 \pm 12.2$ & $90.0 \pm 12.5$ & $<0.0001$ \\
\hline Serum triglycerides $(\mathrm{mmol} / \mathrm{l})$ & $1.3 \pm 0.9$ & $1.3 \pm 0.7$ & $1.3 \pm 0.9$ & $<0.0001$ \\
\hline Serum HDL-cholesterol (mmol/l) & $1.7 \pm 0.5$ & $1.6 \pm 0.5$ & $1.7 \pm 0.5$ & $<0.0001$ \\
\hline Serum LDL-cholesterol (mmol/l) & $3.2 \pm 0.8$ & $3.1 \pm 0.8$ & $3.2 \pm 0.8$ & 0.0610 \\
\hline Fasting plasma glucose (mmol/l) & $5.6 \pm 1.1$ & $5.7 \pm 1.2$ & $5.5 \pm 1.1$ & $<0.0001$ \\
\hline Blood hemoglobin A1c (mmol/mol) & $38.6 \pm 7.2$ & $40.4 \pm 6.5$ & $38.4 \pm 7.3$ & $<0.0001$ \\
\hline Blood urea nitrogen (mmol/l) & $5.2 \pm 2.0$ & $6.9 \pm 3.6$ & $4.9 \pm 1.2$ & $<0.0001$ \\
\hline Serum creatinine $(\mu \mathrm{mol} / \mathrm{l})$ & $66.8 \pm 15.7$ & $90.7 \pm 19.6$ & $63.9 \pm 13.1$ & $<0.0001$ \\
\hline eGFR (ml/min/1.73 m²) & $77.4 \pm 15.3$ & $52.7 \pm 6.8$ & $80.3 \pm 13.2$ & $<0.0001$ \\
\hline Serum uric acid $(\mu \mathrm{mol} / \mathrm{l})$ & $325.2 \pm 85.0$ & $370.0 \pm 89.6$ & $319.8 \pm 82.7$ & $<0.0001$ \\
\hline Serum C-reactive protein $(\mu \mathrm{g} / \mathrm{l})$ & $970.0 \pm 3476.4$ & $1185.4 \pm 3263.3$ & $949.6 \pm 3496.0$ & 0.3496 \\
\hline White blood cells $\left(10^{3} / \mu \mathrm{l}\right)$ & $5.4 \pm 1.6$ & $5.4 \pm 1.6$ & $5.4 \pm 1.6$ & 0.8500 \\
\hline Red blood cells $\left(10^{4} / \mu 1\right)$ & $437.0 \pm 43.6$ & $421.7 \pm 49.1$ & $438.5 \pm 42.8$ & 0.8500 \\
\hline Hemoglobin $(\mathrm{g} / \mathrm{l})$ & $138.1 \pm 15.0$ & $135.2 \pm 16.8$ & $138.4 \pm 14.9$ & $<0.0001$ \\
\hline Hematocrit $(\%)$ & $40.3 \pm 4.2$ & $39.5 \pm 4.5$ & $40.4 \pm 4.1$ & $<0.0001$ \\
\hline Platelet counts $\left(10^{4} / \mu 1\right)$ & $22.4 \pm 5.3$ & $20.6 \pm 5.2$ & $22.5 \pm 5.4$ & $<0.0001$ \\
\hline
\end{tabular}

Data are presented as mean \pm standard deviation or percentages. BP, blood pressure; HDL, high-density lipoprotein; LDL, low-density lipoprotein; eGFR, estimated glomerular filtration rate $\left(\mathrm{ml} / \mathrm{min} / 1.73 \mathrm{~m}^{2}\right)=194 \mathrm{x}$ [age (years) $]^{-0.287} \mathrm{x}$ [serum creatinine $\left.(\mathrm{mg} / \mathrm{dl})\right]^{-1.094} \mathrm{x}[0.739 \mathrm{for}$ females]. Based on Bonferroni's correction, $\mathrm{P}<0.0023$ (0.05/22 tests) was considered to indicate a statistically significant difference and significant values are presented in bold.

Table II. Longitudinal analysis of the associations between eGFR and clinical parameters using the generalized linear mixed-effect model with adjustments for age and gender.

\begin{tabular}{|c|c|c|c|}
\hline Parameter & All & Low eGFR & Control \\
\hline Systolic BP & 0.0780 & 0.5370 & 0.6650 \\
\hline Diastolic BP & 0.6800 & 0.4500 & 0.0110 \\
\hline Mean BP & 0.3000 & 0.4600 & 0.0024 \\
\hline Serum triglycerides & 0.0014 & 0.5200 & 0.1900 \\
\hline Serum HDL-cholesterol & $5.1 \times 10^{-5}$ & $1.6 \times 10^{-5}$ & 0.8400 \\
\hline Serum LDL-cholesterol & $1.7 \times 10^{-11}$ & 1.0000 & 0.5405 \\
\hline Blood hemoglobin A1c & $1.8 \times 10^{-6}$ & 0.5791 & 0.2410 \\
\hline Fasting plasma glucose & $4.2 \times 10^{-7}$ & 0.0035 & 0.0367 \\
\hline Serum uric acid & $<2.0 \times 10^{-16}$ & 0.5600 & $<2.0 \times 10^{-16}$ \\
\hline Body mass index & $1.3 \times 10^{-10}$ & 0.5600 & $2.0 \times 10^{-15}$ \\
\hline
\end{tabular}

BP, blood pressure; HDL, high-density lipoprotein; LDL, low-density lipoprotein; eGFR, estimated glomerular filtration rate $\left(\mathrm{ml} / \mathrm{min} / 1.73 \mathrm{~m}^{2}\right)=194 \mathrm{x}$ [age (years) $]^{-0.287} \mathrm{x}$ [serum creatinine $\left.(\mathrm{mg} / \mathrm{dl})\right]^{-1.094} \mathrm{x}[0.739$ for females]. Based on Bonferroni's correction, $\mathrm{P}<0.0017$ $(0.05 / 30$ tests $)$ was considered to indicate a statistically significant difference and significant values are presented in bold.

subjects. Similar analysis indicated that eGFR was significantly associated with serum concentrations of HDL-cholesterol in subjects with low eGFR, and to serum concentrations of uric acid and BMI in the control group (Table II). Systolic (Fig. 2A), diastolic (Fig. 2B), and mean BP (Fig. 2C), serum concentrations of triglycerides (Fig. 2D) and uric acid (Fig. 2I), FPG level 
Table III. Longitudinal analysis of the associations between eGFR and clinical conditions using the generalized estimating equation with adjustments for age and gender.

\begin{tabular}{|c|c|c|c|}
\hline Parameter & All & Low eGFR & Control \\
\hline Hypertension & 0.0006 & $5.1 \times 10^{-12}$ & 0.1413 \\
\hline Type 2 diabetes mellitus & $<2.0 \times 10^{-16}$ & $1.6 \times 10^{-11}$ & $<2.0 \times 10^{-16}$ \\
\hline Hypertriglyceridemia & 0.1800 & $5.2 \times 10^{-7}$ & 0.2290 \\
\hline Hypo-HDL-cholesterolemia & $<2.0 \times 10^{-16}$ & 0.0034 & 0.0025 \\
\hline Hyper-LDL-cholesterolemia & 0.0182 & 0.0912 & $2.8 \times 10^{-12}$ \\
\hline Hyperuricemia & $<2.0 \times 10^{-16}$ & $<2.0 \times 10^{-16}$ & $<2.0 \times 10^{-16}$ \\
\hline Obesity & $5.5 \times 10^{-10}$ & 0.5065 & $<2.0 \times 10^{-16}$ \\
\hline
\end{tabular}

HDL, high-density lipoprotein; LDL, low-density lipoprotein; eGFR, estimated glomerular filtration rate $\left(\mathrm{ml} / \mathrm{min} / 1.73 \mathrm{~m}{ }^{2}\right)=194 \mathrm{x}[$ age (years) $]^{-0.287} \mathrm{x}$ [serum creatinine $\left.(\mathrm{mg} / \mathrm{dl})\right]^{-1.094} \mathrm{x}[0.739$ for females]. Based on Bonferroni's correction, $\mathrm{P}<0.0024$ (0.05/21 tests) was considered to indicate a statistically significant difference and significant values are presented in bold.

Table IV. Longitudinal analysis of the associations between clinical parameters and age using the generalized linear mixed-effect model with adjustment for gender.

\begin{tabular}{|c|c|c|}
\hline Parameter & Low eGFR & Control \\
\hline Systolic BP & 0.0097 & $<2.0 \times 10^{-16}$ \\
\hline Diastolic BP & $1.6 \times 10^{-8}$ & $<2.0 \times 10^{-16}$ \\
\hline Mean BP & $1.7 \times 10^{-6}$ & $<2.0 \times 10^{-16}$ \\
\hline Serum triglycerides & 0.1760 & $<2.0 \times 10^{-16}$ \\
\hline Serum HDL-cholesterol & 0.2200 & 0.0002 \\
\hline Serum LDL-cholesterol & 0.4100 & $<2.0 \times 10^{-16}$ \\
\hline Blood hemoglobin A1c & $2.8 \times 10^{-9}$ & $<2.0 \times 10^{-16}$ \\
\hline Fasting plasma glucose & 0.0420 & $<2.0 \times 10^{-16}$ \\
\hline Serum uric acid & 0.2100 & $9.2 \times 10^{-8}$ \\
\hline Body mass index & 0.0004 & $<2.0 \times 10^{-16}$ \\
\hline
\end{tabular}

eGFR, estimated glomerular filtration rate; BP, blood pressure; HDL, high-density lipoprotein; LDL, low-density lipoprotein. Based on Bonferroni's correction, $\mathrm{P}<0.0025$ ( $0.05 / 20$ tests) was considered to indicate a statistically significant difference and significant values are presented in bold.

(Fig. 2G), blood hemoglobin A1c content (Fig. 2H) and BMI (Fig. 2J) decreased curvilinearly. However, the serum concentrations of HDL-cholesterol (Fig. 2E) increased as eGFR increased. Serum concentrations of LDL-cholesterol (Fig. 2F) reduced slightly with increases in eGFR.

Longitudinal analysis with the generalized estimating equation following adjustment for age and gender demonstrated a significant association $(\mathrm{P}<0.0024)$ between eGFR and the prevalence of hypertension, type $2 \mathrm{DM}$, hypo-HDL-cholesterolemia, hyperuricemia and obesity based on analysis of data from all subjects. Similar analysis demonstrated a significant association between eGFR and prevalence of hypertension, type $2 \mathrm{DM}$, hypertriglyceridemia and hyperuricemia in subjects with low eGFR, and with type 2 DM, hyper-LDL-cholesterolemia, hyperuricemia and obesity in the control group (Table III).
Table V. Longitudinal analysis of the associations between clinical conditions and age using the generalized estimating equation with adjustment for gender.

\begin{tabular}{lcc}
\hline Parameter & Low eGFR & Control \\
\hline Hypertension & 0.0350 & $\mathbf{< . 0 \times 1 0}-\mathbf{1 6}$ \\
Type 2 diabetes mellitus & 0.9300 & 0.7600 \\
Hypertriglyceridemia & 0.0760 & $\mathbf{< . 0 \times 1 0}$ \\
Hypo-HDL-cholesterolemia & 0.8140 & 0.0068 \\
Hyper-LDL-cholesterolemia & 0.0126 & 0.8400 \\
Hyperuricemia & 0.5600 & $\mathbf{0 . 0 0 3 2}$ \\
Obesity & 0.7630 & 0.2400 \\
\hline
\end{tabular}

eGFR, estimated glomerular filtration rate; HDL, high-density lipoprotein; LDL, low-density lipoprotein. Based on Bonferroni's correction, $\mathrm{P}<0.0036(0.05 / 14$ tests) was considered to indicate a statistically significant difference and significant values are presented in bold.

The prevalence of hypertension (Fig. 3A), hypertriglyceridemia (Fig. 3C) and hyperuricemia (Fig. 3F) decreased as eGFR increased. The prevalence of type 2 DM (Fig. 3B), hypo-HDL-cholesterolemia (Fig. 3D), hyper-LDL-cholesterolemia (Fig. 3E), and obesity (Fig. 3G) demonstrated slight decreases as eGFR increased.

Longitudinal analysis of the associations between clinical parameters and age. Longitudinal analysis was then performed for the associations between various clinical parameters and age in subjects with low eGFR and the control subjects using the generalized linear mixed-effect model (Table IV). The analysis showed significant association of diastolic and mean BP, blood hemoglobin A1c content, and BMI $(\mathrm{P}<0.0025)$ with age among the subjects with low eGFR. In the control group, systolic and mean BP, blood hemoglobin A1c content, and FPG level were correlated positively with age. Conversely, diastolic BP, serum concentrations of triglycerides, HDL- and LDL-cholesterol, uric acid and BMI were negatively correlated with age. 


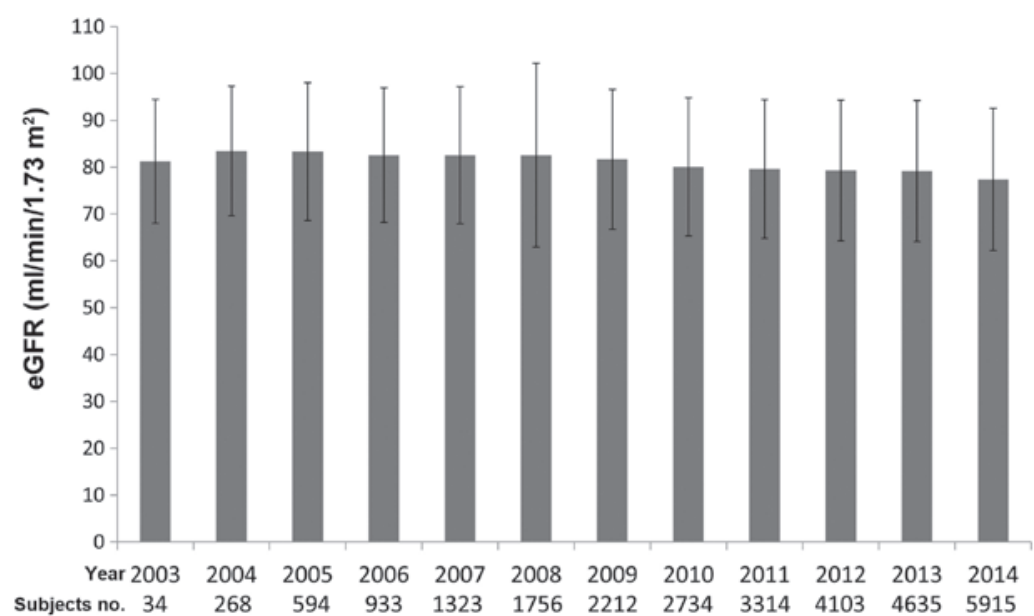

Figure 1. Distribution of eGFR of study subjects. Values are presented as mean \pm standard deviation. eGFR, estimated glomerular filtration rate.

A

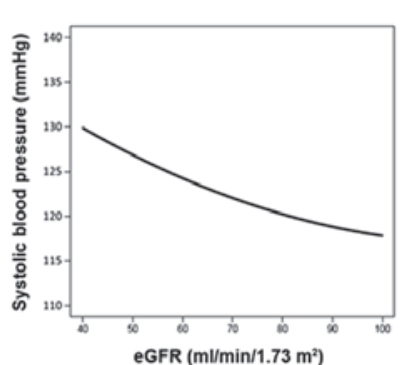

E

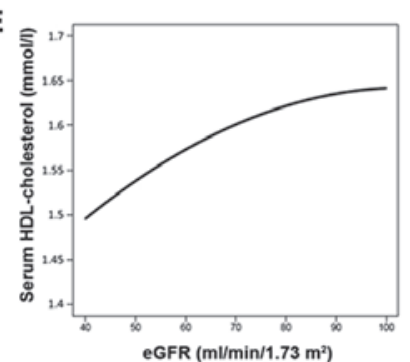

B

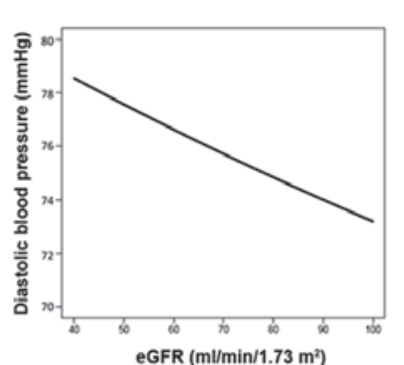

$\mathbf{F}$

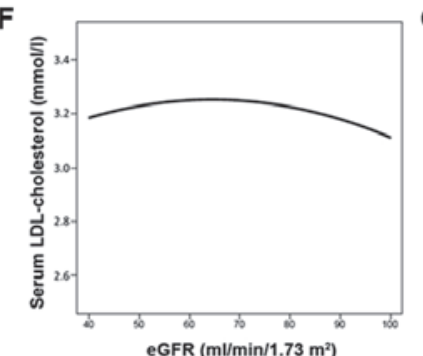

I

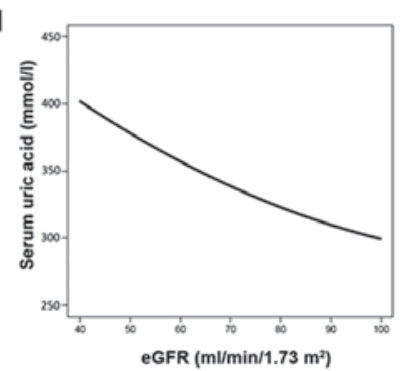

C

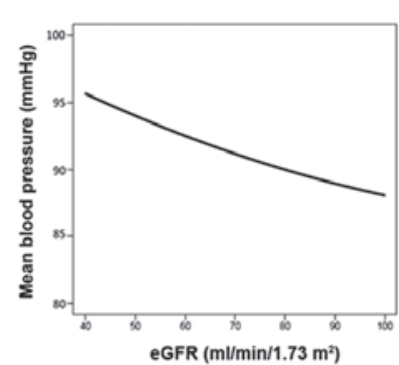

G

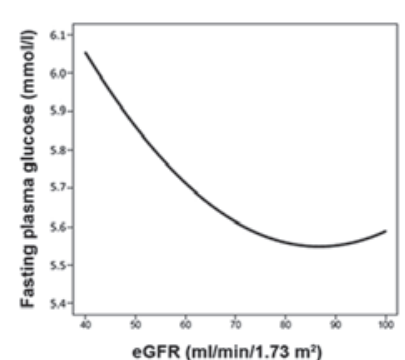

J

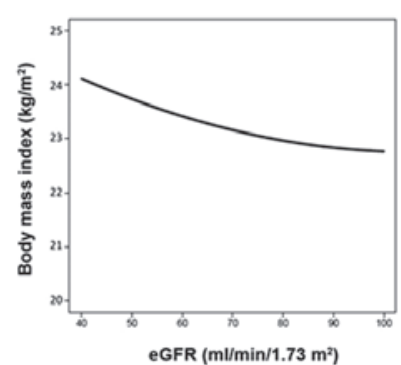

D
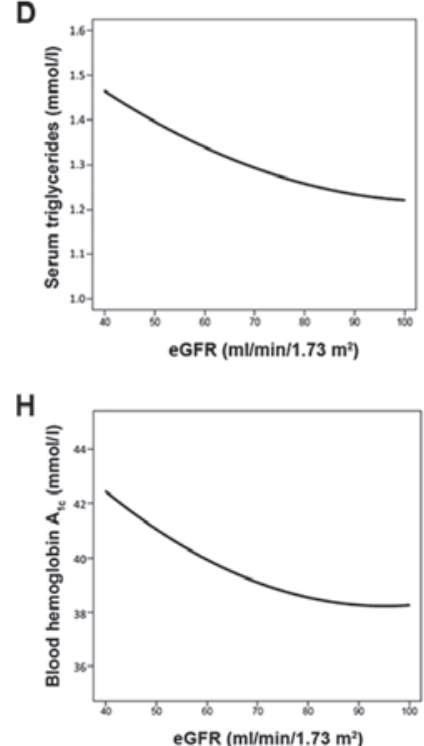

Figure 2. P-values of the association of eGFR with clinical parameters were analyzed by the generalized linear mixed-effect model. Analysis of the association of eGFR with (A) systolic, (B) diastolic and (C) mean blood pressure; serum concentrations of (D) triglycerides, (E) HDL-cholesterol and (F) LDL-cholesterol; (G) fasting plasma glucose level; (H) blood hemoglobin A1c content; (I) serum concentrations of uric acid and (J) body mass index was performed. eGFR, estimated glomerular filtration rate; HDL, high-density lipoprotein; LDL, low-density lipoprotein.

Fig. 4 demonstrates age-associated changes in various clinical parameters. Systolic BP increased with age in the two groups (Fig. 4A). Diastolic BP increased with age, up to $\sim 50$ and 60 years of age, in the subjects with low eGFR and the control subjects, respectively and decreased thereafter (Fig. 4B). Mean BP increased with age up to $\sim 60$ years and $\sim 70$ years in the subjects with low eGFR and the control subjects, respectively and decreased thereafter (Fig. 4C). Serum concentrations of triglycerides (Fig. 4D) and LDL-cholesterol (Fig. 4F) increased with age with a peak at 60 years and decreased thereafter in the control, while they decreased consistently with age in subjects with low eGFR. Serum concentrations of HDL-cholesterol slightly increased with age up to $\sim 50$ years and $\sim 60$ years in the low eGFR group and the control subjects, respectively and decreased thereafter (Fig. 4E). FPG level (Fig. 4G) and blood hemoglobin Alc content (Fig. 4H) increased with age, with a 
A
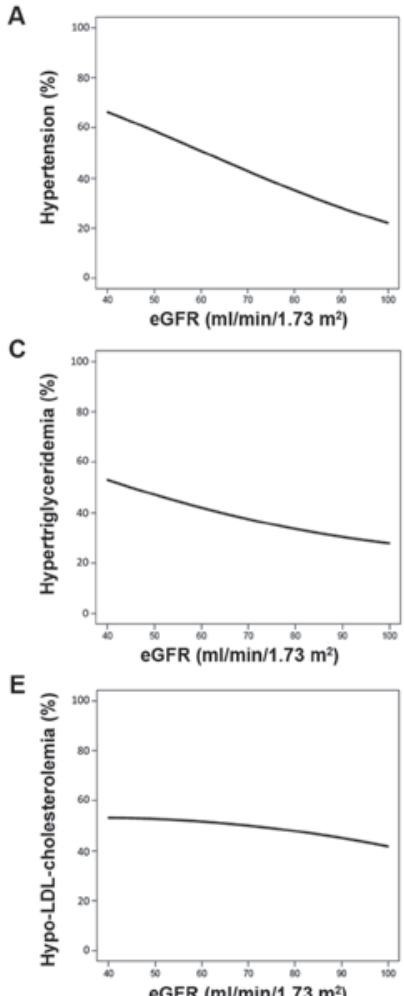

G

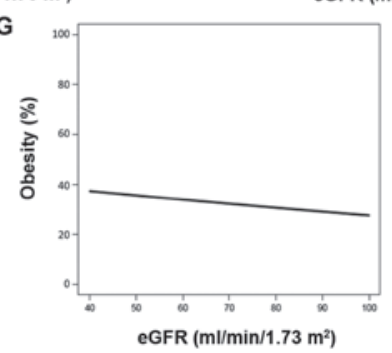

Figure 3. P-values of the association of eGFR with clinical conditions using the generalized estimating equation. The association of eGFR with the prevalence of (A) hypertension, (B) type 2 diabetes mellitus, (C) hypertriglyceridemia, (D) hypo-HDL-cholesterolemia, (E) hyper-LDL-cholesterolemia, (F) hyperuricemia and (G) obesity was analyzed. eGFR, estimated glomerular filtration rate; HDL, high-density lipoprotein; LDL, low-density lipoprotein.

peak at 70 years and decreased thereafter in subjects with low eGFR and in the control subjects. Uric acid decreased gradually with age (Fig. 4I), while BMI increased with age with a peak at 60 years and decreased thereafter, in the two groups (Fig. 4J).

Finally, longitudinal analysis of the associations between clinical conditions and age was conducted using the generalized estimating equation. The analysis demonstrated significant associations between hypertension, hypertriglyceridemia and hyperuricemia $(\mathrm{P}<0.0036)$ and age in the control subjects (Table V). Fig. 5 demonstrates the age-associated changes in the prevalence of clinical conditions. The prevalence of hypertension increased with age in the two groups (Fig. 5A). The prevalence of type $2 \mathrm{DM}$ increased gradually with age in the two groups (Fig. 5B). The prevalence of hypertriglyceridemia increased with age in the control subjects, whereas it decreased with age in the subjects from the low eGFR group (Fig. 5C). The prevalence of hyper-LDL-cholesterolemia decreased with age among subjects with low eGFR, whereas it increased with age in the control subjects (Fig. 5E). The prevalence of hyperuricemia decreased marginally in the two groups (Fig. 5F). The prevalence of hypo-HDL-cholesterolemia and obesity showed no association with age (Fig. 5D and G).

\section{Discussion}

The present study examined the association between renal dysfunction, and various parameters and clinical status in a longitudinal epidemiological study. The results indicated that impairment of renal function had detrimental effects on various clinical parameters and patient health status.

Hypertension is a frequent complication in patients with renal impairment, and is present concurrently with other factors involved in the development of diabetes and/or non-diabetic CKD progression $(8,17,18)$. The pathophysiology and mechanisms of hypertension in renal impairment are complex. Previous studies reported that activation of the sympathetic nervous system and renin-angiotensin-aldosterone system contributes to the development of hypertension in individuals with CKD (19). Furthermore, sodium retention, volume expansion, and reduced synthesis of vasodilator substances subsequent to the rise in BP may exacerbate hypertension. Impairment of autoregulation of glomerular pressure may also lead to glomerular hypertension (20). Given that activation of the renin-angiotensin-aldosterone system not only causes a rise in $\mathrm{BP}$, but also promotes cell proliferation, inflammation, and matrix accumulation, it may also lead to the progression of CKD (17). Therefore, strict control of BP is highly recommended for CKD subjects and is expected to delay renal injury progression. The current results of eGFR correlating significantly and negatively with the prevalence of hypertension in the low eGFR group are consistent with the results of previous studies $(8,17,18)$, although the exact underlying mechanisms were not evaluated.

In the Framingham Heart Study (21), systolic BP increased with age from 30 to 84 years, whereas diastolic BP increased until the fifth decade, and subsequently slowly decreased from the ages of 60 to 84 years. Similar age-associated changes in BP parameters were observed in the present study. Although a significant association between eGFR and systolic, diastolic or mean BP was not identified, each BP parameter was greater in the low eGFR group (even in young individuals; aged 40-60 years), and each BP parameter peak occurred earlier in the low eGFR group, when compared with the control subjects.

Dyslipidemia is also common in patients with CKD (22). Weiner and Sarnak (23) reported high prevalence of hyper-LDL-cholesterolemia $(\sim 85 \%)$ among non-dialysis nephrotic CKD patients, together with high incidences of hypertriglyceridemia and hypo-HDL-cholesterolemia. The possible underlying mechanism of dyslipidemia in CKD patients may be reflected in apolipoprotein profiles, and lipid profiles vary depending on the level of renal function and the degree of proteinuria (22). Non-dialysis CKD patients, particularly those with nephrotic syndrome, exhibit decreased activity of total and tissue-bound lipoprotein lipase, which is the predominant enzyme involved in the catabolism of triglycerides, resulting in high triglycerides levels. In addition, reduced synthesis of apoprotein A-I and HDL-cholesterol, which correlates with the decline in GFR, is observed in all spectra of CKD patients (24). However, serum concentrations of total and LDL-cholesterol 
A

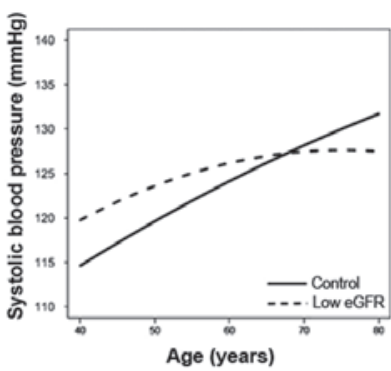

E

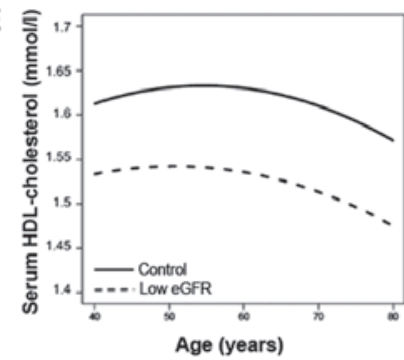

B
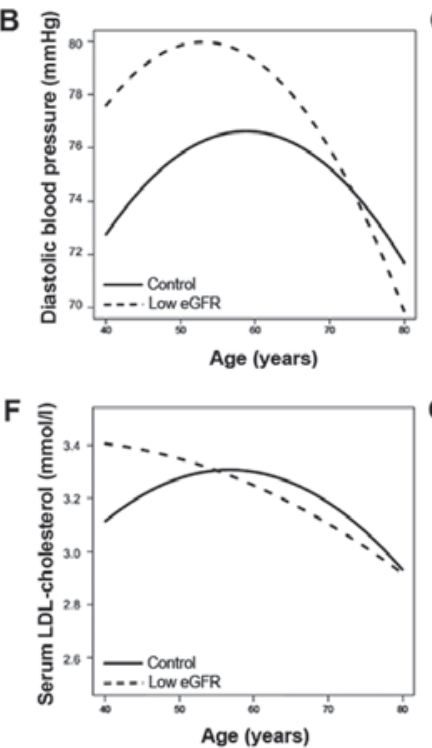

'

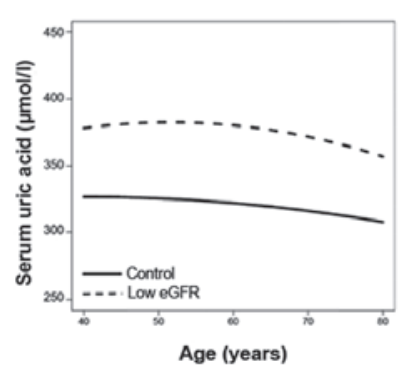

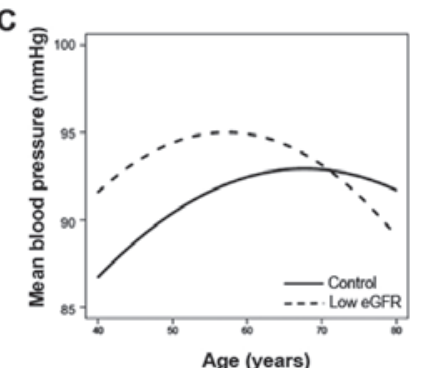

Age (years)
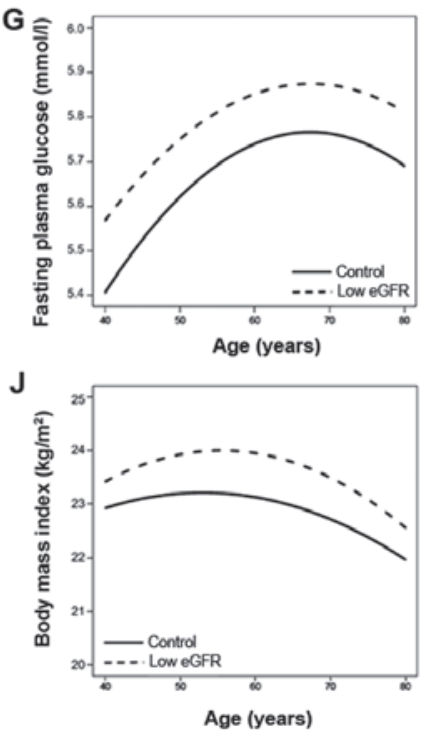

D
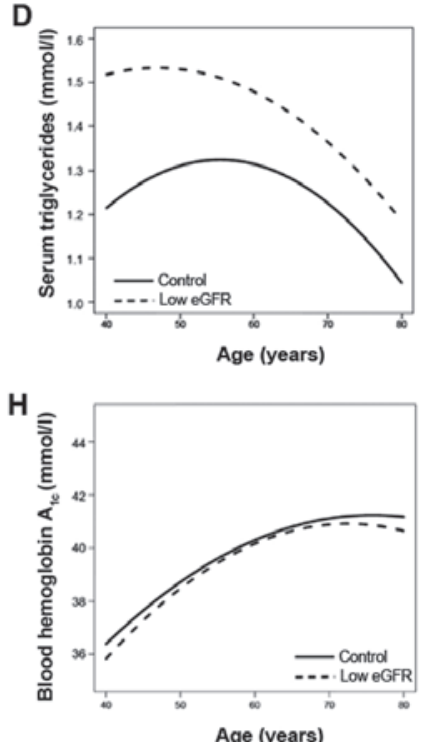

Figure 4. P-values of the association of various clinical parameters with age using the generalized linear mixed-effect model in individuals with low eGFR and control subjects. The association of age with (A) systolic, (B) diastolic and (C) mean blood pressure; serum concentrations of (D) triglycerides, (E) HDL-cholesterol and (F) LDL-cholesterol; (G) fasting blood glucose level; (H) blood hemoglobin A1c content; (I) serum concentrations of uric acid and (J) body mass index. eGFR, estimated glomerular filtration rate; HDL, high-density lipoprotein; LDL, low-density lipoprotein.
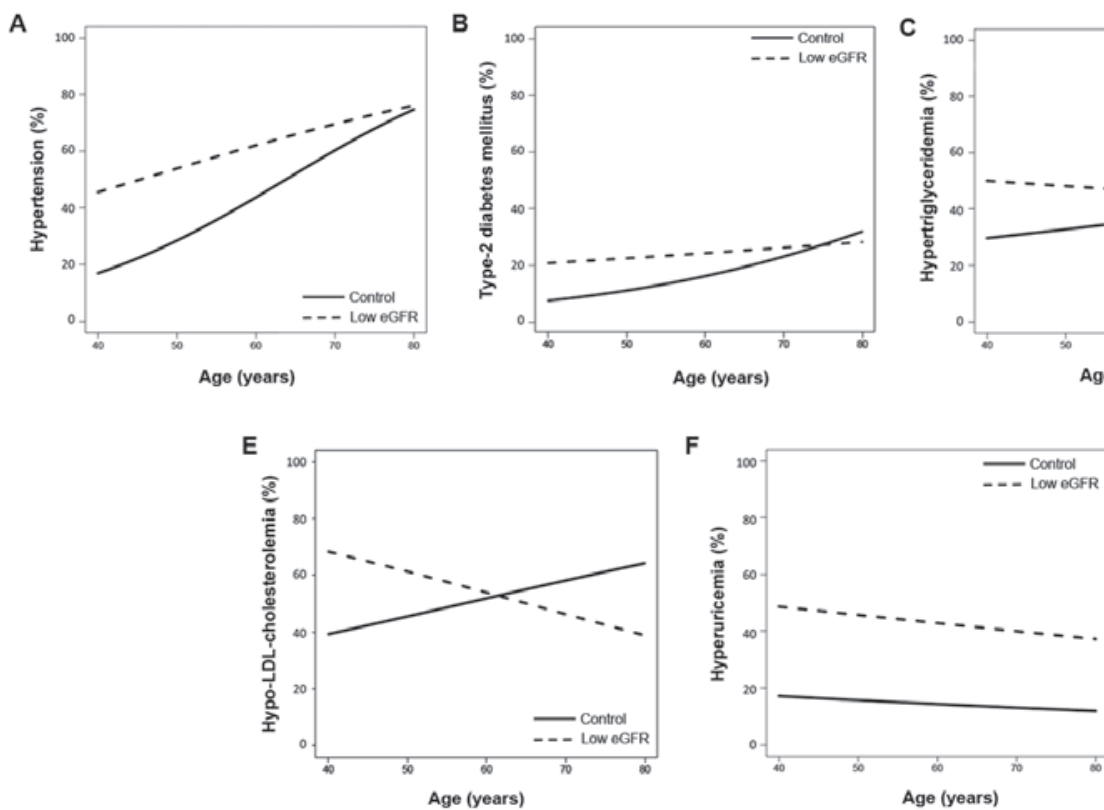
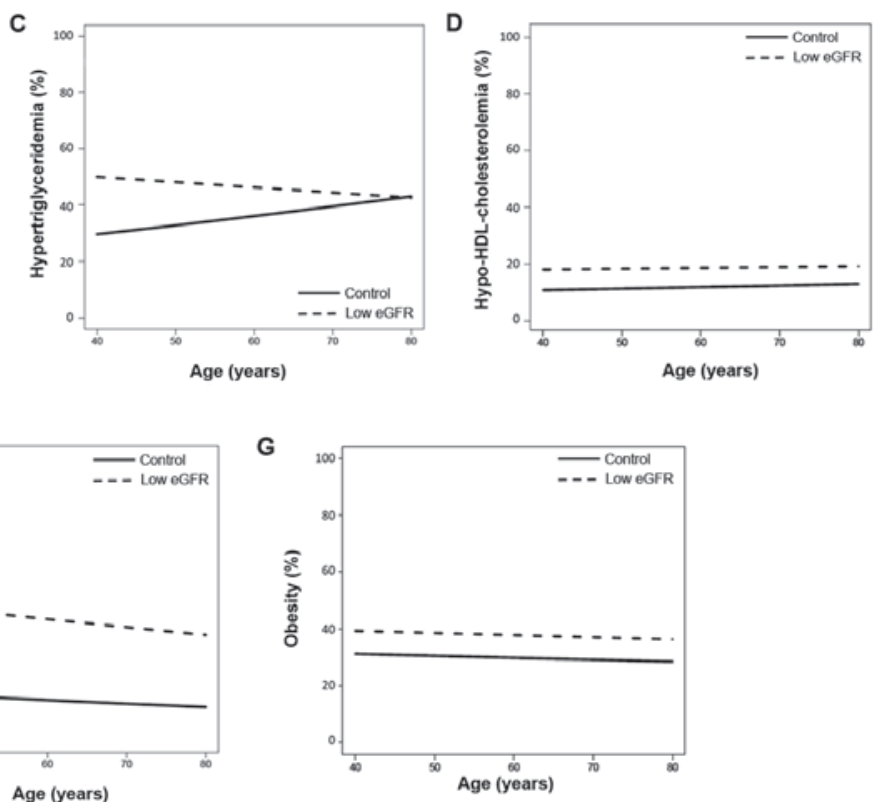

Figure 5. P-values of the association of various clinical conditions with age in subjects with low eGFR and the control subjects. The association of age with the prevalence of (A) hypertension, (B) type 2 diabetes mellitus, (C) hypertriglyceridemia, (D) hypo-HDL-cholesterolemia, (E) hyper-LDL-cholesterolemia, (F) hyperuricemia and (G) obesity. eGFR, estimated glomerular filtration rate; HDL, high-density lipoprotein; LDL, low-density lipoprotein. 
decrease as CKD progresses to kidney failure (23). The current results demonstrated that eGFR correlated inversely with hypertriglyceridemia, and positively with serum HDL-cholesterol in individuals with low eGFR. In addition, eGFR was observed to correlate inversely with hyper-LDL-cholesterolemia in the control subjects. These results were consistent with a previous report (25), showing a causal association with early and advanced stages of renal dysfunction.

Dyslipidemia coupled with renal dysfunction enhances the development of atherosclerosis and cardiovascular diseases (1). Atherosclerosis of renal arteries and subsequent damage to marginal cells accelerates renal dysfunction (26). Previous studies demonstrated that statin therapy reduce the decline in eGFR in non-dialysis CKD patients $(27,28)$; therefore, early detection and intensive treatment are essential for these patients. The current study found that eGFR was significantly associated with the prevalence of dyslipidemia, even in control subjects and in younger individuals, indicating that aggressive clinical management is required in such populations.

In the present study, although eGFR correlated negatively with the prevalence of type 2 DM in subjects with low eGFR and the control subjects, no such correlation was identified with FPG level or blood hemoglobin Alc content. This discrepancy between our results and previous studies (29) may be due to treatment of type $2 \mathrm{DM}$. Renal dysfunction promotes insulin resistance in patients with mild to moderate stage of CKD, even when eGFR is maintained within the normal range (30). In addition, insulin resistance is associated with increased risk for CKD and is an independent predictor of cardiovascular diseases (30). Therefore, annual screening for type $2 \mathrm{DM}$ is recommended for all CKD subjects (31).

Glucose intolerance increases progressively with age. Glucose intolerance in the elderly may be due to impaired insulin secretion, increased insulin resistance or changes in the endocrine system, obesity, physical inactivity, reduced dietary carbohydrate, impaired renal function or administration of certain therapeutic agents $(32,33)$. The age associated increases in fasting blood glucose level and blood hemoglobin A1c content observed in the present study are consistent with previous observations (34).

Hyperuricemia is common in CKD patients, and the association between uric acid and worsening renal function is well established (35). Animal studies have shown that oxidative stress and endothelial dysfunction due to hyperuricemia cause renal damage (36). Furthermore, one epidemiological study suggested that increases in serum uric acid levels precede the development of CKD (37). In the present study, eGFR correlated significantly and negatively with serum uric acid level and the prevalence of hyperuricemia in control individuals. These findings are consistent with those of a previous study (37). Inhibition of uric acid excretion due to renal dysfunction may account for this association, although the underlying molecular mechanism remains unclear.

The prevalence of obesity is increasing worldwide, and is a risk factor of numerous diseases, including CKD (38). Insulin resistance, hyperinsulinemia, abnormal levels of inflammatory adipokines, and the activated renin-angiotensin-aldosterone system may contribute to the development of glomerular injury (39). Furthermore, obesity and CKD may synergistically affect the occurrence of cardiovascular diseases (40). In the present study, eGFR correlated negatively with BMI and the prevalence of obesity in control subjects. Further studies are required to examine the importance of BMI control in the prevention of future renal function loss.

There were certain limitations of the present study. First, given that we have not performed a replication study, validation of the current findings will require additional, independent subject panels. In addition, although the mean follow-up period was 5 years, the period was heterogeneous and varied from 1 to 11 years. Furthermore, the number of subjects varied between the different age groups. The present study also included subjects who were being treated for hypertension, type 2 DM, dyslipidemia and/or hyperuricemia. Finally, information regarding the underlying renal disease in each patient with low eGFR was not evaluated. Such information could be obtained by detailed clinical examination, including renal biopsy; however, such diagnostic procedure was not considered to be feasible for a population-based epidemiological study.

In conclusion, the results of the present study indicated that low eGFR has detrimental effects on various clinical parameters and conditions, resulting in increased risk of hypertension, dyslipidemia, type 2 DM, hyperuricemia and obesity.

\section{Acknowledgements}

The present study was supported by CREST of the Japan Science and Technology Agent (to Professor Yoshiji Yamada and Professor Ichiro Takeuchi) as well as by JSPS KAKENHI (grant no. JP15H04772 to Y.Y.).

\section{References}

1. National Kidney Foundation: K/DOQI clinical practice guidelines for chronic kidney disease: Evaluation, classification, and stratification. Am J Kidney Dis 39 (Suppl 1): S1-S266, 2002.

2. Sugiyama H, Yokoyama H, Sato H, Saito T, Kohda Y, Nishi S, Tsuruya K, Kiyomoto H, Iida H, Sasaki T, et al; Committee for Standardization of Renal Pathological Diagnosis; Committee for Kidney Disease Registry; Japanese Society of Nephrology: Japan Renal Biopsy Registry and Japan Kidney Disease Registry: Committee Report for 2009 and 2010. Clin Exp Nephrol 17: 155-173, 2013.

3. Culleton BF, Larson MG, Wilson PW, Evans JC, Parfrey PS and Levy D: Cardiovascular disease and mortality in a community-based cohort with mild renal insufficiency. Kidney Int 56: 2214-2219, 1999.

4. Mann JF: Cardiovascular risk in patients with mild renal insufficiency: Implications for the use of ACE inhibitors. Presse Med 34: 1303-1308, 2005.

5. Fox CS, Larson MG, Leip EP, Meigs JB, Wilson PW and Levy D: Glycemic status and development of kidney disease: The Framingham Heart Study. Diabetes Care 28: 2436-2440, 2005.

6. Selvin E, Ning Y, Steffes MW, Bash LD, Klein R, Wong TY, Astor BC, Sharrett AR, Brancati FL and Coresh J: Glycated hemoglobin and the risk of kidney disease and retinopathy in adults with and without diabetes. Diabetes 60: 298-305, 2011.

7. Moorhead JF, Chan MK, El-Nahas M and Varghese Z: Lipid nephrotoxicity in chronic progressive glomerular and tubulo-interstitial disease. Lancet 2: 1309-1311, 1982.

8. Bakris GL, Williams M, Dworkin L, Elliott WJ, Epstein M, Toto R, Tuttle K, Douglas J, Hsueh W and Sowers J; National Kidney Foundation Hypertension and Diabetes Executive Committees Working Group: Preserving renal function in adults with hypertension and diabetes: A consensus approach. Am J Kidney Dis 36: 646-661, 2000.

9. Yamada Y, Matsui K, Takeuchi I, Oguri M and Fujimaki T: Association of genetic variants with hypertension in a longitudinal population-based genetic epidemiological study. Int $\mathrm{J}$ Mol Med 35: 1189-1198, 2015. 
10. Yamada Y, Matsui K, Takeuchi I, Oguri M and Fujimaki T: Association of genetic variants of the $\alpha$-kinase 1 gene with type 2 diabetes mellitus in a longitudinal population-based genetic epidemiological study. Biomed Rep 3: 347-354, 2015.

11. Yamada Y, Matsui K, Takeuchi I and Fujimaki T: Association of genetic variants with dyslipidemia and chronic kidney disease in a longitudinal population-based genetic epidemiological study. Int J Mol Med 35: 1290-1300, 2015.

12. Yamada Y, Matsui K, Takeuchi I and Fujimaki T: Association of genetic variants with coronary artery disease and ischemic stroke in a longitudinal population-based genetic epidemiological study. Biomed Rep 3: 413-419, 2015.

13. Matsuo S, Imai E, Horio M, Yasuda Y, Tomita K, Nitta K, Yamagata K, Tomino Y, Yokoyama $\mathrm{H}$ and Hishida A Collaborators developing the Japanese equation for estimated GFR: Revised equations for estimated GFR from serum creatinine in Japan. Am J Kidney Dis 53: 982-992, 2009.

14. Kanazawa M, Yoshiike N, Osaka T, Numba Y, Zimmet P and Inoue S: Criteria and classification of obesity in Japan and Asia-Oceania. Asia Pac J Clin Nutr 11 (s8): S732-S737, 2002.

15. Cheng J, Edwards LJ, Maldonado-Molina MM, Komro KA and Muller KE: Real longitudinal data analysis for real people: Building a good enough mixed model. Stat Med 29: 504-520, 2010.

16. Gibbons RD, Hedeker D and DuToit S: Advances in analysis of longitudinal data. Annu Rev Clin Psychol 6: 79-107, 2010.

17. Maschio G, Oldrizzi L, Marcantoni C and Rugiu C: Hypertension and progression of renal disease. J Nephrol 13: 225-227, 2000

18. Staessen JA, Gasowski J, Wang JG, Thijs L, Den Hond E, Boissel JP, Coope J, Ekbom T, Gueyffier F, Liu L, et al: Risks of untreated and treated isolated systolic hypertension in the elderly: Meta-analysis of outcome trials. Lancet 355: 865-872, 2000.

19. Taal MW and Brenner BM: Renoprotective benefits of RAS inhibition: From ACEI to angiotensin II antagonists. Kidney Int 57: 1803-1817, 2000.

20. Ljutić D and Kes P: The role of arterial hypertension in the progression of non-diabetic glomerular diseases. Nephrol Dial Transplant 18 (Suppl 5): v28-v30, 2003.

21. Franklin SS: Ageing and hypertension: The assessment of blood pressure indices in predicting coronary heart disease. J Hypertens Suppl 17: S29-S36, 1999.

22. Kasiske BL: Hyperlipidemia in patients with chronic renal disease. Am J Kidney Dis 32 (Suppl 3): S142-S156, 1998.

23. Weiner DE and Sarnak MJ: Managing dyslipidemia in chronic kidney disease. J Gen Intern Med 19: 1045-1052, 2004.

24. Hirano T, Furukawa S, Kurokawa M, Ebara T, Dixon JL and Nagano S: Intracellular apoprotein B degradation is suppressed by decreased albumin concentration in Hep G2 cells. Kidney Int 47: 421-431, 1995.

25. Thompson M, Ray U, Yu R, Hudspeth A, Smillie M, Jordan N and Bartle J: Kidney Function as a Determinant of HDL and Triglyceride Concentrations in the Australian Population. J Clin Med 5: 35, 2016

26. Schaeffner ES, Kurth T, Curhan GC, Glynn RJ, Rexrode KM, Baigent C, Buring JE and Gaziano JM: Cholesterol and the risk of renal dysfunction in apparently healthy men. J Am Soc Nephrol 14: 2084-2091, 2003.
27. Sandhu S, Wiebe N, Fried LF and Tonelli M: Statins for improving renal outcomes: A meta-analysis. J Am Soc Nephrol 17: 2006-2016, 2006.

28. Su X, Zhang L, Lv J, Wang J, Hou W, Xie X and Zhang H: Effect of statins on kidney disease outcomes: A systematic review and meta-analysis. Am J Kidney Dis 67: 881-892, 2016.

29. Lin CC, Chen CC, Chen FN, Li CI, Liu CS, Lin WY, Yang SY, Lee CC and Li TC: Risks of diabetic nephropathy with variation in hemoglobin A1c and fasting plasma glucose. Am J Med 126: 1017.e1-1017.e10, 2013.

30. Liao MT, Sung CC, Hung KC, Wu CC, Lo L and Lu KC: Insulin resistance in patients with chronic kidney disease. $\mathrm{J}$ Biomed Biotechnol 2012: 691369, 2012.

31. National Kidney Foundation: KDOQI Clinical Practice Guideline for Diabetes and CKD: 2012 Update. Am J Kidney Dis 60: 850-886, 2012.

32. Chang AM and Halter JB: Aging and insulin secretion. Am J Physiol Endocrinol Metab 284: E7-E12, 2003.

33. Stout RW: Glucose tolerance and ageing. J R Soc Med 87: 608-609, 1994

34. Shimokata H, Muller DC, Fleg JL, Sorkin J, Ziemba AW and Andres R: Age as independent determinant of glucose tolerance. Diabetes 40: 44-51, 1991.

35. Nacak H, van Diepen $M$, de Goeji MC, Rotmans JI and Dekker FW; PREPARE-2 study group: Uric acid: association with rate of renal function decline and time until start of dialysis in incident pre-dialysis in incident pre-dialysis patients. BMC Nephrol 15: 91, 2014.

36. Mazzali M, Hughes J, Kim YG, Jefferson JA, Kang DH, Gordon KL, Lan HY, Kivlighn S and Johnson RJ: Elevated uric acid increases blood pressure in the rat by a novel crystal-independent mechanism. Hypertension 38: 1101-1106, 2001.

37. Bo S, Cavallo-Perin P, Gentile L, Repetti E and Pagano G: Hypouricemia and hyperuricemia in type 2 diabetes: Two different phenotypes. Eur J Clin Invest 31: 318-321, 2001.

38. Fox CS, Larson MG, Leip EP, Culleton B, Wilson PW and Levy D: Predictors of new-onset kidney disease in a community-based population. JAMA 291: 844-850, 2004

39. Cusumano AM, Bodkin NL, Hansen BC, Iotti R, Owens J, Klotman PE and Kopp JB: Glomerular hypertrophy is associated with hyperinsulinemia and precedes overt diabetes in aging rhesus monkeys. Am J Kidney Dis 40: 1075-1085, 2002.

40. Beddhu S, Kimmel PL, Ramkumar $\mathrm{N}$ and Cheung AK: Associations of metabolic syndrome with inflammation in CKD: Results From the Third National Health and Nutrition Examination Survey (NHANES III). Am J Kidney Dis 46: 577-586, 2005 